\title{
Lorentzian Threads as Gatelines and Holographic Complexity
}

\author{
Juan F. Pedraza $\odot,{ }^{1,2}$ Andrea Russo $\odot,{ }^{1}$ Andrew Svesko $\odot,{ }^{1}$ and Zachary Weller-Davies $\odot^{1}$ \\ ${ }^{1}$ Department of Physics and Astronomy, University College London, London WC1E 6BT, United Kingdom \\ ${ }^{2}$ Martin Fisher School of Physics, Brandeis University, Waltham, Massachusetts 02453, USA
}

(Received 16 July 2021; revised 21 October 2021; accepted 15 November 2021; published 30 December 2021)

\begin{abstract}
The continuous min flow-max cut principle is used to reformulate the "complexity = volume" conjecture using Lorentzian flows-divergenceless norm-bounded timelike vector fields whose minimum flux through a boundary subregion is equal to the volume of the homologous maximal bulk Cauchy slice. The nesting property is used to show the rate of complexity is bounded below by "conditional complexity," describing a multistep optimization with intermediate and final target states. Conceptually, discretized Lorentzian flows are interpreted in terms of threads or gatelines such that complexity is equal to the minimum number of gatelines used to prepare a conformal field theory (CFT) state by an optimal tensor network (TN) discretizing the state. We propose a refined measure of complexity, capturing the role of suboptimal TNs, as an ensemble average. The bulk symplectic potential provides a "canonical" thread configuration characterizing perturbations around arbitrary CFT states. Its consistency requires the bulk to obey linearized Einstein's equations, which are shown to be equivalent to the holographic first law of complexity, thereby advocating a notion of "spacetime complexity."
\end{abstract}

DOI: 10.1103/PhysRevLett.127.271602

Introduction.-Gravity has an information theoretic character. The sharpest realization of this is captured by the Ryu-Takayanagi (RT) formula [1,2], relating the area of minimal codimension-2 surfaces $m(A)$ in a $(d+1)$ dimensional (bulk) anti-de Sitter (AdS) spacetime to the entanglement entropy (EE) $S(A)$ of a conformal field theory (CFT) state restricted to a $(d-1)$-dimensional boundary subregion $A$ homologous to $m$. The RT formula generalizes Bekenstein-Hawking black hole entropy and satisfies all known properties of the von Neumann entropy. More strikingly, it was used to show gravitational field equations are dual to the first law of entanglement $[3,4]$, encapsulating the slogan "entanglement = geometry" [5-7].

Recently, the RT prescription was reformulated in terms of flows or holographic "bit threads" [8], where $m(A)$ is replaced by the maximum flux of a divergenceless normbounded Riemannian vector field $v$ through $A$,

$S(A)=\max _{v \in \mathcal{F}} \int_{A} v, \quad \mathcal{F} \equiv\left\{v|\nabla \cdot v=0| v \mid, \leq \frac{1}{4 G_{N}}\right\}$

The equivalence between the two follows from the max flow-min cut theorem, a known principle in network theory, where the "min cut" is the minimal surface, and was proven

Published by the American Physical Society under the terms of the Creative Commons Attribution 4.0 International license. Further distribution of this work must maintain attribution to the author(s) and the published article's title, journal citation, and DOI. Funded by SCOAP ${ }^{3}$. using convex optimization techniques [9]. It has several generalizations and applications, e.g., [10-18]. Not only does (1) have technical advantages, it offers conceptual insight: a thread emanating from $A$ is interpreted as a channel carrying a single (qu)bit encoding the microstate of $A$, where the maximum number of threads gives $S(A)$, which may be distilled as Bell pairs. Bit threads have also led to insights into tensor network (TN) models of spacetime, e.g., $[19,20]$, and the emergence of gravity via the closedness of the "canonical" flow solution [15].

Entanglement alone, however, does not describe all aspects of bulk gravitational physics [21]. In particular, the late time growth of the Einstein-Rosen bridge inside eternal black holes is not captured by entanglement, but rather complexity. By complexity, one typically means the state complexity, i.e., the smallest number of unitary operators (gates) needed to obtain a particular final state from a given initial state. While the definition of state complexity in a field theory remains an active area of investigation (cf. [22-24]), two proposals for its geometric interpretation have emerged: "complexity = volume" $(\mathrm{CV})$ [25-28] and "complexity = action" (CA) [29-31]. The $\mathrm{CV}$ conjecture says the complexity $\mathcal{C}$ of a CFT state defined on a Cauchy slice $\sigma_{A}$ delimiting a boundary region $A$, so that $\partial A=\sigma_{A}$, is dual to the volume of an extremal codimension- 1 bulk hypersurface $\Sigma$ homologous $A$

$$
\mathcal{C}\left(\sigma_{A}\right)=\frac{1}{G_{N} \ell} \max _{\Sigma \sim A} \operatorname{vol}[\Sigma(A)] .
$$

Here, $\ell$ is some undetermined bulk length scale, e.g., the AdS curvature, and the homology condition $\Sigma \sim A$ implies 
$\partial \Sigma=\partial A=\sigma_{A}$. Alternatively, CA equates complexity with the gravitational action $I$ evaluated over the Wheeler-De Witt (WDW) patch. CV and CA are similar qualitatively, however, here we focus on CV duality.

Given their similar geometric character, it is natural to compare the CV proposal (2) to the entropy-area RT prescription. In light of the bit thread reformulation, one may suspect the CV proposal for holographic complexity (2) likewise has a flow based interpretation. Indeed, via the min flow-max cut (MFMC) theorem, where Riemannian flows are replaced by Lorentzian flows, the minimum flux through a boundary region $A$ is equal to the maximum cut of a surface homologous to $A$ [9]. In this Letter we use the continuous MFMC principle [9] to reformulate the CV conjecture of holographic complexity in terms of Lorentzian flows and explore some of their properties and implications. We provide a more detailed account and additional results, including explicit geometric realizations of Lorentzian flows in [32].

$\mathrm{CV}$ and the min flow-max cut theorem.-The continuous version of the MFMC theorem was first presented and proved in [9]. It says the minimum flux of a Lorentzian flow $v$ through a boundary region $A$ of a compact Lorentzian manifold $\mathcal{M}$ is equal to the volume $V$ of the maximal bulk codimension-1 Cauchy slice $\Sigma \sim A$ :

$\min _{v} \int_{A} v=\alpha \max _{\Sigma \sim A}(V(\Sigma)), \quad \int_{A} v \equiv \int_{A} \sqrt{h} n_{\mu} v^{\mu}$,

where $\alpha \in \mathbb{R}^{+}, n_{\mu}$ is a unit normal covector to $A$, and $\sqrt{h}$ is the induced volume element. The flow $v$ is a timelike vector field obeying,

$$
\nabla \cdot v=0, \quad v^{0}>0, \quad|v| \geq \alpha .
$$

It is now natural to reformulate $\mathrm{CV}$ duality (2) in terms of Lorentzian flows. Precisely, upon setting $\alpha=\left(1 / G_{N} \ell\right)$, we propose $\mathcal{C}$ is the minimum flux of a divergenceless normbounded timelike vector field $v$ through $A$

$\mathcal{C}\left(\sigma_{A}\right)=\min _{v \in \mathcal{F}} \int_{A} v, \quad \mathcal{F} \equiv\left\{v|\nabla \cdot v=0| v \mid, \geq \frac{1}{G_{N} \ell}\right\}$.

Via the MFMC theorem, Eq. (5) is equal to the maximal volume of a Cauchy slice $\Sigma$ homologous to $A$ (3).

Properties of Lorentzian flows. - An important lemma to MFMC is the nesting property. Concretely, consider two nested boundary regions $A B$ and $A B \supset A, B \equiv A B \backslash A$, in a compact, oriented Lorentzian manifold $\mathcal{M}$, where $A$ lies to the future of $B, A>B$. That is, the boundary is foliated by slices $\sigma_{A}>\sigma_{A B}$. Assuming $\mathcal{M}$ obeys the strong energy condition this foliation induces a foliation of the bulk by nonintersecting maximal cuts $\Sigma(A)>\Sigma(A B)$ [33]. Nesting tells us there exists a flow $v(A, A B)$ which simultaneously minimizes flux through $A$ and $A B$. Equivalently, $v(A, A B)$ maximizes the flux through $B$, conditioned on minimizing flux through $A B$.
The nesting property uncovers a number of interesting behaviors holographic complexity must satisfy. First, when there are two nested regions as above, one has

$$
\mathcal{C}\left(\sigma_{A}\right)-\mathcal{C}\left(\sigma_{A B}\right)=-\int_{B} v(A, A B) .
$$

Since a flow $v(A B)$ with minimal flux through $A B$ has generally less flux than $v(A, A B)$ through $B$, we find

$$
\mathcal{C}\left(\sigma_{A}\right)-\mathcal{C}\left(\sigma_{A B}\right) \leq \mathcal{C}\left(\sigma_{A} \mid \sigma_{A B}\right),
$$

where we have defined $\mathcal{C}\left(\sigma_{A} \mid \sigma_{A B}\right) \equiv-\min \int_{B} v(A B)$.

Since $\mathcal{C}\left(\sigma_{A B}\right)$ is the complexity of a state at time $t_{A B}=t_{A}-\delta t$, from (6) we find the rate $\dot{\mathcal{C}}$ in terms of maximal flux through $B$. By the momentum-volumecomplexity (PVC) relation [34-36], we deduce

$$
-\dot{\mathcal{C}}=\lim _{B \rightarrow 0} \frac{1}{\delta t} \int_{B} v(A, A B)=\int_{\Sigma} T_{\mu \nu} n^{\mu} \zeta^{\nu}-R_{\Sigma} .
$$

The first term in (8) is the integrated momentum flux $P_{\zeta}$, where $n$ is the future-pointing unit-normal to $\Sigma$ and $\zeta$ is an "infalling" vector tangent to $\Sigma$ asymptotically equal to a radial, inward-pointing vector with modulus given by the radius of the sphere at infinity. The remainder $R_{\Sigma}$ arises from integrating the momentum constraint and vanishes when $\zeta$ is a conformal Killing vector, in which case the maximal flux through $B$ is only given by $P_{\zeta}$.

Generalizing to three nested boundary regions $A, A B$, and $A B C$, with $\Sigma(A)>\Sigma(A B)>\Sigma(A B C)$, we uncover the following relationship between the minimal flux $\Phi(X)$ through each region $X$ and complexity $\mathcal{C}\left(\sigma_{A B C}\right)$

$$
\Phi(A C)+\Phi(B C)-\Phi(C) \leq \mathcal{C}\left(\sigma_{A B C}\right) .
$$

This is the Lorentzian analog of the strong subadditivity of EE. Moreover, in the limit $B, C$ shrink, together with (8), we recover $\ddot{\mathcal{C}}=\dot{P}_{\zeta}[36,37]$, suggesting Newton's laws of gravitation have an origin in complexity.

CV complexity is also known to obey a superadditivity property [38-40], defined in terms of subregion complexity $C_{S}\left(\sigma_{X}\right)$ [41], where $\sigma_{X} \subset \sigma_{A}$ is a boundary spatial subregion. Let $R$ be a Hubeny-Rangamani-Takayanagi (HRT) surface subdividing $\sigma_{A}=\sigma_{X} \cup \sigma_{Y}$. We bipartition $A=$ $A_{X} \cup A_{Y}$ with $A_{X} \cap A_{Y}=\emptyset$. The MFMC theorem allows us to reformulate superadditivity as

$$
C_{S}\left(\sigma_{X} \cup \sigma_{Y}\right) \geq C_{S}\left(\sigma_{X}\right)+C_{S}\left(\sigma_{Y}\right),
$$

where $C_{S}\left(\sigma_{X}\right) \leq \int_{A_{X}} v(A)$ and similarly for $C_{S}\left(\sigma_{Y}\right)$.

Interpretation: "gatelines" and tensor networks.Similar to the bit thread interpretation of Riemannian flows [8], there is a unique mapping between Lorentzian flows and what we call "Lorentzian threads" or gatelines [9]. Specifically, threads are defined as the integral lines of the flows $v$ (4) of transverse density $|v|$. Denoting $N_{A}$ as the number of threads passing through the maximal slice $\Sigma(A)$, given (5), CV complexity is understood as the minimum number of threads passing through $\Sigma(A)$, 

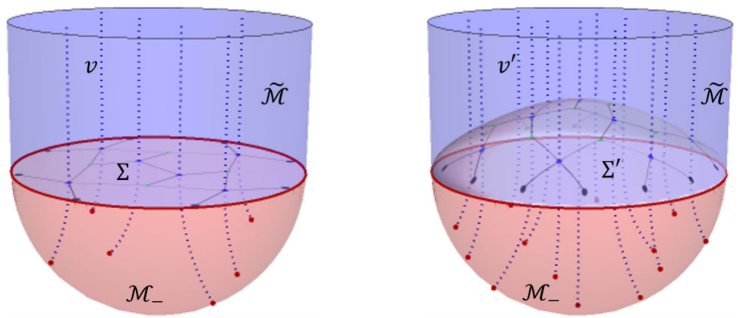

FIG. 1. Complexity is equal to the minimum number of gatelines preparing a state on maximal volume slice $\Sigma$. Optimal flow prepares optimal TN (left); suboptimal flows prepare more complex suboptimal TNs (right).

$$
\mathcal{C}\left(\sigma_{A}\right)=\min N_{A} .
$$

This observation suggests threads prepare the state on $\Sigma(A)$ from a specific reference CFT state defined on the infinite past of the manifold. More precisely, recall bulk Lorentzian spacetimes describe time evolution of CFT states prepared by Euclidean path integrals with sources turned on [42-46]. A reference state is specified on a bulk Cauchy slice $\Sigma_{-}$of the southern hemisphere of Euclidean AdS, such that for generic analytic initial data the bulk Einstein's equations reveal which sources are used to prepare the state [47]. The length of the Lorentzian cylinder glued at $\Sigma_{-}$then gives the duration of evolution (Fig. 2).

Threads flowing into $A$ enter from the Euclidean submanifold attached to boundary sources and pass through $\Sigma(A)$. The minimal flux configuration optimally prepares the CFT state on $\Sigma(A)$, i.e., requiring fewer operations to assemble the state. Thus, Lorentzian threads act as gatelines: timelike trajectories representing unitary gates needed to transform a reference state to a target state. Consequently, complexity is the minimum number of gatelines through $\Sigma(A)$ preparing the target state:

$\mathcal{C} \sim$ number of threads $\sim$ number of gates to prepare the state.

Conceptually, then, $\mathcal{C}\left(\sigma_{A} \mid \sigma_{A B}\right)$ in (7) is the minimum number of gatelines needed to prepare a state on $\Sigma(A)$ given the state prepared on $\Sigma(A B)$. That is, $\mathcal{C}\left(\sigma_{A} \mid \sigma_{A B}\right)$ is the "conditional complexity," describing a two step optimization, first preparing the intermediate state $\Sigma(A B)$ before preparing $\Sigma(A)$. Vanishing flux through $B$ implies the same number of gatelines prepare states on $\Sigma(A B)$ and $\Sigma(A)$, thereby having equal complexity. Meanwhile, when $\mathcal{C}\left(\sigma_{A}\right)>\mathcal{C}\left(\sigma_{A B}\right)$, for example, flux through $B$ provides additional gatelines to prepare $\Sigma(A)$.

The gateline interpretation deepens our insight into TN constructions of spacetimes $[19,20]$. TNs act as discretizations of bulk spatial slices, where the EE is computed by counting cuts along the TN, and complexity equals the number of tensors that describes the TN. Combining this prescription for complexity with (12), it is then natural to conjecture an optimal thread configuration $v$ prepares the
TN on $\Sigma$. We imagine attaching a unitary to each thread, connecting to each physical tensor of the network, so number of threads $\sim$ number of tensors (Fig. 1). These unitaries act similar to disentanglers in a MERA TN [48], transforming a reference state to its target. Upon analytic continuation, this operation generates time evolution and the $\mathrm{TN}$ acts as a quantum circuit.

Canonical flows from the bulk symplectic form.-To characterize perturbative excited states, e.g., linear perturbations to vacuum AdS, we develop a notion of "perturbative Lorentzian threads." Analogous to [15], for a perturbed metric of the form $g_{\mu \nu}^{\eta}=g_{\mu \nu}+\eta \delta g_{\mu \nu}$, with $\eta$ small, we define $v_{\eta}=v+\eta \delta v+\mathcal{O}\left(\eta^{2}\right)$, where $v_{\eta}$ obeys the flow criteria (4), constraining $\delta v$. Thus, given a metric $g_{\mu \nu}$ and a solution $v$ to the unperturbed min flow problem, we can solve for the minimizing flow $v_{\eta}$.

It is convenient to work with differential forms. We exploit the map between divergenceless vector fields $v$ and closed $(D-1)$ forms $u$ in a $D$-dimensional background

$$
u=\frac{1}{(D-1) !} \epsilon_{\mu_{1}, \ldots, \mu_{D-1} \nu} v^{\nu} d x^{\mu_{1}} \wedge \cdots \wedge d x^{\mu_{D-1}},
$$

where $\epsilon=\sqrt{-g} \varepsilon$ is the volume form on the manifold. Criteria (4) apply to forms $u$, and we rewrite CV (5) as

$$
\mathcal{C}\left(\sigma_{A}\right)=\min _{u} \int_{A} u \text {. }
$$

For metrics perturbatively close to $g_{\mu \nu}$, denote the perturbed $(D-1)$ form by $u^{\eta}=u+\eta \delta u$. Divergencelessness, the norm bound, and restriction to $\Sigma$ translate to

$$
\begin{aligned}
& d(u+\eta \delta u)=0 \Rightarrow d(\delta u)=0, \\
& -\langle u, u\rangle_{g}+\eta\left[2\langle u, \delta u\rangle_{g}+\langle u, u\rangle_{\delta g}\right] \geq 1, \\
& \left.(u+\eta \delta u)\right|_{\Sigma}=\tilde{\epsilon}+\left.\eta \delta \tilde{\epsilon} \Rightarrow \delta u\right|_{\Sigma}=\delta \tilde{\epsilon},
\end{aligned}
$$

with $\langle u, u\rangle_{g} \equiv[1 /(D-1) !] g^{\mu_{1} \nu_{1}}, \ldots, g^{\mu_{D-1} \nu_{D-1}} u_{\mu_{1}, \ldots, \mu_{D-1}} \times$ $u_{\nu_{1}, \ldots, \nu_{D-1}}$, and $\tilde{\epsilon}$ is the pullback of $\epsilon$ to $\Sigma$. Hence, when studying linear perturbations of complexity around a background we must find a closed $(D-1)$ form $\delta u$ satisfying (15).

A canonical choice for $\delta u$ is the bulk Lorentzian symplectic current $\omega_{\text {bulk }}^{L}$. This follows from the equivalence between the boundary symplectic form $\Omega_{B}$ and the bulk symplectic form $[49,50]$

$$
\Omega_{B}\left(\delta_{1} \tilde{\lambda}, \delta_{2} \tilde{\lambda}\right)=i \int_{\partial \mathcal{M}_{-}} \omega_{\text {bulk }}^{E}\left(\phi, \delta_{1} \phi, \delta_{2} \phi\right) .
$$

Precisely, for holographic CFT states prepared by Euclidean path integrals with sources $\lambda$ [45], the space of sources defines a Kähler manifold whose Kähler 2 form $\Omega_{B}$ is determined by the bulk Euclidean action. One then invokes the extrapolate dictionary to relate sources $\lambda$ to bulk fields $\phi$, and the variation of the $D$-dimensional Lagrangian form $\delta \mathbf{L}=-E_{\phi} \delta \phi+d \theta(\phi, \delta \phi)$. Here, $E_{\phi}$ is a $D$ form 
characterizing the equations of motion for $\phi$, which are assumed to be satisfied, $E_{\phi}=0$, and $\theta$ is the symplectic potential, whose variation gives the symplectic current $\omega_{\text {bulk }}\left(\phi ; \delta_{1} \phi, \delta_{2} \phi\right)=\delta_{1} \theta\left(\phi, \delta_{2} \phi\right)-\delta_{2} \theta\left(\phi, \delta_{1} \phi\right)$.

If $\delta_{1,2} \phi$ obey the linearized equations of motion, $\delta_{1,2} E_{\phi}=0$ and $d \omega_{\text {bulk }}=0$, so the southern hemisphere $\partial \mathcal{M}_{-}$can be pushed to an initial value surface $\Sigma$, replacing $\omega_{\text {bulk }}^{E}$ with its Lorentzian counterpart $\omega_{\text {bulk }}^{L}$

$$
\Omega_{B}\left(\delta_{1} \tilde{\lambda}, \delta_{2} \tilde{\lambda}\right)=\int_{\Sigma} \omega_{\text {bulk }}^{L}\left(\phi, \delta_{1} \phi, \delta_{2} \phi\right) .
$$

When the boundary sources are deformed by the "new York" transformation $\delta_{Y}$, Eq. (3.11) in [51], the bulk symplectic form $\Omega_{\text {bulk }} \equiv \int_{\Sigma} \omega_{\text {bulk }}$ is identified with the variation of the volume of the maximal bulk slice $\Sigma[49,50]$,

$$
\Omega_{B}\left(\delta_{Y} \tilde{\lambda}, \delta \tilde{\lambda}\right)=\Omega_{\text {bulk }}\left(\delta_{Y} \phi, \delta \phi\right)=\frac{(d-1) \tilde{\alpha}}{8 \pi G_{N}} \delta V,
$$

with $\tilde{\alpha}$ some constant. Foliating the bulk by constant-time surfaces $\Sigma$ with spatial metric $h_{i j}$ and extrinsic curvature $K_{i j}$, in terms of $\delta_{Y}$, the Hamiltonian constraint of general relativity $\mathcal{H}$ reads, $\delta_{Y} \mathcal{H}=2(d-2) K$. This is satisfied when trace of the extrinsic curvature $K=0$. Thence, $\delta_{Y}$ is on shell when $\Sigma$ is a maximal surface.

For $\tilde{\alpha} \equiv[8 \pi / \ell(d-1)]$, Eq. (18) naturally proposes a notion of varying complexity, $\delta \mathcal{C}[50]$. In fact, defining complexity as an integral of kinetic energy over the space of sources, $\mathcal{C}\left(s_{i}, s_{f}\right) \equiv \int_{s_{i}}^{s_{f}} d s g_{a b} \dot{\lambda}^{a} \dot{\lambda}^{b}$, with $s$ parametrizing trajectories in this space, $\delta \mathcal{C}$ obeys a first law

$$
\delta_{\lambda_{f}} \mathcal{C}=\Omega_{B}\left(\delta_{Y} \tilde{\lambda}, \delta \tilde{\lambda}\right)
$$

This is a boundary relation. For holographic CFTs, via (17) and (18) one has a first law of (CV) complexity.

Returning to the definition of perturbative thread form $u$ (15), it is straightforward to verify $\omega_{\text {bulk }}^{L}\left(\delta_{Y}, \delta\right)$ satisfies the conditions on $\delta u$. Thus $\omega_{\text {bulk }}^{L}\left(\delta_{Y}, \delta\right)$ represents a "canonical" thread configuration, solves the MFMC program and is closed for on-shell perturbations.

First law of complexity and Einstein's equations.-From (19) we can derive the covariant linearized Einstein's equations, differing from [52]. Our method is similar to $[3,4]$ using the first law of EE. While the following holds for general bulk states, here we consider vacuum perturbations, hence $\phi$ only represents the bulk metric.

Applying Stokes' theorem and using $E_{\phi}=0$, we have

$$
i \int_{\mathcal{M}_{-}} d \omega_{\text {bulk }}^{E}=\Omega_{B}\left(\delta_{Y} \tilde{\lambda}, \delta \tilde{\lambda}\right)-\delta V .
$$

Assuming the holographic version of the first law (19), the right hand side vanishes, requiring $d \omega_{\text {bulk }}^{E}\left(\delta_{Y}, \delta\right)=0$ for arbitrary variations $\delta$. Since $\delta_{Y}$ is a diffeomorphism for perturbations around vacuum $\mathrm{AdS}$, then

$$
d \omega_{\text {bulk }}^{E}\left(\delta_{Y}, \delta\right)=-\epsilon \delta E^{\mu \nu} \delta_{Y} g_{\mu \nu}=0,
$$

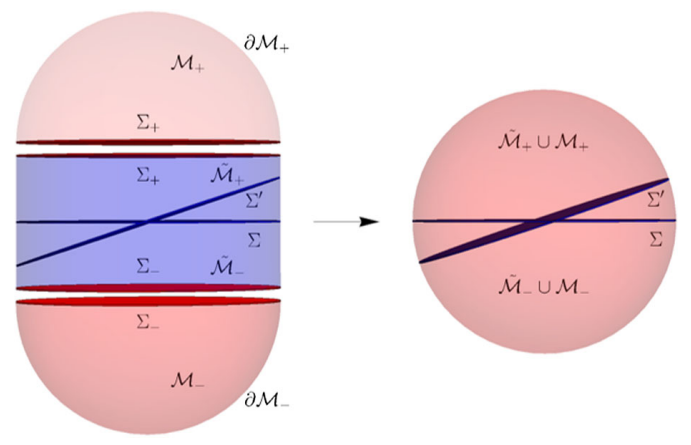

FIG. 2. We pick a different bulk Lorentzian slice $\Sigma^{\prime}$ to partition the two regions, in particular $\Sigma^{\prime}$ could be the constant time surface of a Lorentz boosted observer.

where $\delta E^{\mu \nu}=(1 / \sqrt{g})\left(\delta S_{\text {grav }} / \delta g_{\mu \nu}\right)$, is no longer a $d$ form on $\mathcal{M}$.

We now argue demanding (21) for all Lorentzian initial data is equivalent to the linearized Einstein's equations $\delta E^{\mu \nu}=0$ being satisfied everywhere in the bulk $\mathcal{M}$. First consider a maximal slice $\Sigma$ along which the southern and northern hemispheres are glued. In Euclidean Poincaré coordinates one has

$\tau^{2} \delta E^{\tau \tau}+2 \tau z \delta E^{\tau z}+\left(\tau^{2}+z^{2}\right) \delta E^{i i}+\tau z^{2} \delta E^{z z}=0$.

We now demand this holds for all maximal slices $\Sigma$, each providing data for different Lorentz observers in $\mathcal{M}$, which will allow us to prove $\delta E_{\mu \nu}=0$ everywhere.

We start by deforming the contour to allow for some real time evolution. This is done by gluing a cylinder section $\tilde{\mathcal{M}}$ in between the southern and northern hemispheres, along surfaces $\Sigma_{-}$and $\Sigma_{+}$(Fig. 2). The state on $\Sigma_{-}$is prepared by a Euclidean path integral over $\mathcal{M}_{-}$. It then evolves to $\Sigma_{+}$ and closes at $\mathcal{M}_{+}[42,43]$. Lorentzian $\operatorname{AdS} \tilde{\mathcal{M}}$ is split into sections $\tilde{\mathcal{M}}_{-}, \tilde{\mathcal{M}}_{+}$along $\Sigma$. Performing a Wick rotation on $\tilde{\mathcal{M}}_{ \pm}$, manifolds $\tilde{\mathcal{M}}_{-} \cup \mathcal{M}_{-}$and $\tilde{\mathcal{M}}_{+} \cup \mathcal{M}_{+}$describe state preparation on $\Sigma$. However, there is nothing special about $\Sigma$; we could have chosen another slice $\Sigma^{\prime}$, e.g., a constanttime surface of a Lorentz boosted observer. Wick rotating $\tilde{\mathcal{M}}_{ \pm}^{\prime}$, we have a path integral over the sphere, preparing initial data on $\Sigma^{\prime}$.

If $\Sigma^{\prime}$ is related to $\Sigma$ by an isometry then in the new coordinates the metric will take the same form, as will the initial data on $\Sigma^{\prime}$. Consequently, $\delta_{Y}$ is invariant, $\left(\delta_{Y} g\right)^{\prime}\left[x^{\prime}(x)\right]=\left(\delta_{Y} g\right)(x)$. The equations of motion, however, transform as $\delta E^{\prime \mu \nu}\left[x^{\prime}(x)\right]=\Lambda_{\gamma}^{\mu} \Lambda_{\delta}^{\nu} E^{\gamma \delta}(x)$, where $\Lambda_{\nu}^{\mu}=\left(\partial x^{\prime \mu} / \partial x^{\nu}\right)$. Since $d \omega_{\text {bulk }}^{E}\left(\delta_{Y}, \delta\right)=0$, we deduce

$$
\delta E^{\prime \mu \nu}\left(\delta_{Y} g\right)_{\mu \nu}^{\prime}\left[x^{\prime}(x)\right]=\Lambda_{\rho}^{\mu} \Lambda_{\sigma}^{\nu} \delta E^{\rho \sigma} \delta_{Y} g_{\mu \nu}(x)=0 .
$$

Thus, demanding the constraint (21) holds for all maximal slices $\Sigma$ in different Lorentz frames means

$$
\Lambda_{\rho}^{\mu} \Lambda_{\sigma}^{\nu} \delta E^{\rho \sigma} \delta_{Y} g_{\mu \nu}=0
$$


for any rotation of Euclidean AdS. Together with the Bianchi identities $\nabla_{\mu} \delta E^{\mu \nu}=0$, this is enough to conclude $\delta E_{\mu \nu}=0$ holds everywhere in $\mathcal{M}$. Hence, assuming CV duality, the first law of complexity implies the linearized Einstein's equations around vacuum AdS.

Note we can easily accommodate higher derivative gravities, with the volume replaced by the "generalized volume" [53]. Also, we emphasize the bulk-boundary symplectic form equivalence (16) holds for perturbations over general states, not just around vacuum AdS. Then, as suggested in [54] for EE, any asymptotically AdS spacetime obeying CV duality and the first law should satisfy the full nonlinear Einstein equations. Thus, bulk gravity emerges from boundary complexity.

An ensemble proposal of holographic complexity.-Thus far we have focused on optimal flows. Here, we argue suboptimal flows - those with more flux and prepare (more complex) suboptimal TNs-must play a role in defining holographic complexity beyond CV duality.

We propose a more general prescription using suboptimal TNs. We are partly motivated by the "maximin" prescription [55] for computing EE, a two step algorithm where one picks a slice $\Sigma^{\prime}$, finds the minimal surface $m_{A}^{\prime}$, and then maximizes over all slices. Extrapolating to TNs, one first finds the minimal number of cuts on a TN on a $\Sigma^{\prime}$ and then maximize over all TNs in different $\Sigma^{\prime}$ [56]:

$$
S_{A} \sim \max _{\Sigma^{\prime}} \min [\text { number of cuts]. }
$$

A lesson is drawn from (25): for the computation of EEs, not only the TN on the maximal slice plays a role but also suboptimal TNs defined over other slices. This is particularly crucial for dynamical setups. Thus, a more refined measure of complexity capturing a notion of state independence is one where all TNs are taken into account. In terms of flows, optimal flows $v$ prepare a $\mathrm{TN}$ on the maximal slice while suboptimal flows $v^{\prime}$ (those with higher flux) prepare TNs over different slices $\Sigma^{\prime}$. Thus, an averaged measure of complexity accounting all TNs is alternately given by averaging over suboptimal flows.

For specific states, e.g., static ones, the optimal TN is enough to compute the full set of EEs since the associated RT surfaces all lie on a constant- $t$ maximal slice $\Sigma$, and complexity is its volume. However, for generic out-ofequilibrium settings, $\Sigma$ cannot be foliated by HRT surfaces in general. Consequently, appealing to state independence, we need to consider TNs defined over all possible slices $\Sigma^{\prime}$. Since these TNs have different numbers of tensors and thus different complexities, we must consider an appropriate average over $\Sigma^{\prime}$ to fully characterize the state. Concretely, we should consider an ensemble over all possible TNs defined over all $\Sigma^{\prime}$. Formally,

$$
\mathcal{Z} \sim \int \mathcal{D}\left[\Sigma^{\prime}\right] e^{-\frac{1}{\hbar} \mathcal{S}\left[\Sigma^{\prime}\right]}, \quad \Sigma^{\prime} \in \text { WDW patch },
$$

for a given measure of integration $\mathcal{D}\left[\Sigma^{\prime}\right]$ and weight $\mathcal{S}\left[\Sigma^{\prime}\right]$ we leave unspecified. We introduced a control parameter " $\hbar$ " where small $\hbar$ defines a saddle-point approximation, where the maximal slice $\Sigma$ emerges as a "classical" saddle in the case of static spacetimes, for example, if $\mathcal{S}\left[\Sigma^{\prime}\right] \sim$ $\operatorname{vol}\left[\Sigma^{\prime}\right]$. Last, $\hbar$ is taken to be a covariant parameter which takes different values depending on the background, e.g., $\hbar$ could be a timescale of the state, where for static cases $\hbar \rightarrow 0$, and $\hbar \neq 0$ otherwise.

Assuming Eq. (26), we propose

$$
\mathcal{C} \sim \frac{1}{\mathcal{Z}} \int \mathcal{D}\left[\Sigma^{\prime}\right] \operatorname{vol}\left[\Sigma^{\prime}\right] e^{-(1 / \hbar) \mathcal{S}\left[\Sigma^{\prime}\right]},
$$

for appropriate optimized choices of $\mathcal{S}, \hbar$, and measure of integration. When $\hbar \rightarrow 0$ we recover $\mathrm{CV}$ duality, but generally (27) gives a weighted average deviating from $\mathrm{CV}$. Alternatively, in terms of flows we define an average

$$
v_{\text {avg }} \sim \frac{1}{\mathcal{Z}} \int \mathcal{D}\left[v^{\prime}\right] v^{\prime} e^{-\frac{1}{\hbar} S\left[v^{\prime}\right]},
$$

which obeys $\nabla \cdot v_{\text {avg }}=0$, but relaxes the norm bound.

Discussion.-CV duality reformulated using Lorentzian flows reveals complexity may be interpreted as the minimum number of gatelines needed to prepare an optimal TN discretizing the state, where more complex TNs are prepared by suboptimal flows. To account for generic TNs we propose complexity is to be given by a weighted average over all Cauchy slices in the WDW patch.

Our proposal is similar to the holographic dual of the path integral optimization definition of complexity [24,57], where optimization is equivalent to maximizing an AdS Hartle-Hawking $(\mathrm{HH})$ wave function given by a Euclidean path integral of a bulk gravity action over metrics induced on a codimension-1 probe brane $Q$ of tension $T$ [58,59]. Via a saddle-point analysis, the maximization of the $\mathrm{HH}$ wave function implies $Q$ provides a constant mean curvature (CMC) slicing of empty AdS. The tension provides a measure of the complexity: $T \propto K=0$ the path integral complexity functional is optimized; CMC slices $T \neq 0$ correspond to suboptimal TNs. Both proposals thus make use of suboptimal TNs, and we suspect in some contexts the two will coincide. Particularly, when $Q$ foliates the WDW patch the two proposals may be equal when $\Sigma^{\prime}$ has CMC. Alternatively, Lorentzian path integral complexity was shown to behave as CA duality. It is worth deepening this connection and seeing how it relates to other complexity proposals [60-63].

We are grateful to C. Agón, J. Barbón, E. Caceres, W. Fischler, M. Headrick, M. Heller, T. Jacobson, J. MartínGarcía, R. Myers, M. Sasieta, L. Susskind, T. Takayanagi, and M. Visser for useful correspondence. This work is supported by the Simons Foundation via It from Qubit Collaboration and by EPSRC. 
[1] S. Ryu and T. Takayanagi, Phys. Rev. Lett. 96, 181602 (2006).

[2] V. E. Hubeny, M. Rangamani, and T. Takayanagi, J. High Energy Phys. 07 (2007) 062.

[3] N. Lashkari, M. B. McDermott, and M. Van Raamsdonk, J. High Energy Phys. 04 (2014) 195.

[4] T. Faulkner, M. Guica, T. Hartman, R. C. Myers, and M. Van Raamsdonk, J. High Energy Phys. 03 (2014) 051.

[5] M. Van Raamsdonk, Gen. Relativ. Gravit. 42, 2323 (2010).

[6] E. Bianchi and R. C. Myers, Classical Quantum Gravity 31, 214002 (2014).

[7] V. Balasubramanian, B. D. Chowdhury, B. Czech, and J. de Boer, J. High Energy Phys. 01 (2015) 048.

[8] M. Freedman and M. Headrick, Commun. Math. Phys. 352, 407 (2017).

[9] M. Headrick and V. E. Hubeny, Classical Quantum Gravity 35, 105012 (2018).

[10] J. Harper, M. Headrick, and A. Rolph, J. High Energy Phys. 11 (2018) 168.

[11] S. X. Cui, P. Hayden, T. He, M. Headrick, B. Stoica, and M. Walter, Commun. Math. Phys. 376, 609 (2020).

[12] C. A. Agón, J. De Boer, and J. F. Pedraza, J. High Energy Phys. 05 (2019) 075.

[13] J. Harper and M. Headrick, J. High Energy Phys. 08 (2019) 101.

[14] C. A. Agón and M. Mezei, J. High Energy Phys. 11 (2021) 167.

[15] C. A. Agón, E. Cáceres, and J. F. Pedraza, J. High Energy Phys. 01 (2021) 193.

[16] M. Headrick and V. E. Hubeny (to be published).

[17] C. A. Agón and J. F. Pedraza, arXiv:2105.08063.

[18] A. Rolph, arXiv:2105.08072.

[19] G. Vidal, Phys. Rev. Lett. 99, 220405 (2007).

[20] B. Swingle, Phys. Rev. D 86, 065007 (2012).

[21] L. Susskind, Fortschr. Phys. 64, 49 (2016).

[22] S. Chapman, M. P. Heller, H. Marrochio, and F. Pastawski, Phys. Rev. Lett. 120, 121602 (2018).

[23] R. Jefferson and R. C. Myers, J. High Energy Phys. 10 (2017) 107.

[24] P. Caputa, N. Kundu, M. Miyaji, T. Takayanagi, and K. Watanabe, Phys. Rev. Lett. 119, 071602 (2017).

[25] L. Susskind, Fortschr. Phys. 64, 24 (2016); 64, 44(A) (2016).

[26] L. Susskind and Y. Zhao, arXiv:1408.2823.

[27] D. Stanford and L. Susskind, Phys. Rev. D 90, 126007 (2014).

[28] J. Couch, W. Fischler, and P. H. Nguyen, J. High Energy Phys. 03 (2017) 119.

[29] A. R. Brown, D. A. Roberts, L. Susskind, B. Swingle, and Y. Zhao, Phys. Rev. Lett. 116, 191301 (2016).

[30] A. R. Brown, D. A. Roberts, L. Susskind, B. Swingle, and Y. Zhao, Phys. Rev. D 93, 086006 (2016).

[31] Z.-Y. Fan and M. Guo, J. High Energy Phys. 08 (2018) 031; 09 (2019) 121(E).

[32] J. F. Pedraza, A. Russo, A. Svesko, and Z. Weller-Davies, arXiv:2106.12585.
[33] J. Couch, S. Eccles, T. Jacobson, and P. Nguyen, J. High Energy Phys. 11 (2018) 044.

[34] L. Susskind, arXiv:1802.01198.

[35] J. L. F. Barbon, J. Martin-Garcia, and M. Sasieta, J. High Energy Phys. 04 (2021) 250.

[36] J. L. F. Barbon, J. Martin-Garcia, and M. Sasieta, Phys. Rev. D 102, 101901(R) (2020).

[37] L. Susskind, Front. Phys. 8, 262 (2020).

[38] C. A. Agón, M. Headrick, and B. Swingle, J. High Energy Phys. 02 (2019) 145.

[39] E. Cáceres, J. Couch, S. Eccles, and W. Fischler, Phys. Rev. D 99, 086016 (2019).

[40] E. Caceres, S. Chapman, J. D. Couch, J. P. Hernandez, R. C. Myers, and S.-M. Ruan, J. High Energy Phys. 03 (2020) 012.

[41] See Supplemental Material at http://link.aps.org/supplemental/ 10.1103/PhysRevLett.127.271602 for details.

[42] K. Skenderis and B. C. van Rees, Phys. Rev. Lett. 101, 081601 (2008).

[43] K. Skenderis and B. C. van Rees, J. High Energy Phys. 05 (2009) 085.

[44] M. Botta-Cantcheff, P. Martínez, and G. A. Silva, J. High Energy Phys. 02 (2016) 171.

[45] D. Marolf, O. Parrikar, C. Rabideau, A. Izadi Rad, and M. Van Raamsdonk, J. High Energy Phys. 06 (2018) 077.

[46] M. Botta-Cantcheff, P. J. Martínez, and G. A. Silva, J. High Energy Phys. 04 (2019) 028.

[47] A. Belin and B. Withers, J. High Energy Phys. 12 (2020) 185.

[48] G. Vidal, Phys. Rev. Lett. 101, 110501 (2008).

[49] A. Belin, A. Lewkowycz, and G. Sárosi, Phys. Lett. B 789, 71 (2019).

[50] A. Belin, A. Lewkowycz, and G. Sárosi, J. High Energy Phys. 03 (2019) 044.

[51] J. W. York, Jr., Phys. Rev. Lett. 28, 1082 (1972).

[52] B. Czech, Phys. Rev. Lett. 120, 031601 (2018).

[53] P. Bueno, V. S. Min, A. J. Speranza, and M. R. Visser, Phys. Rev. D 95, 046003 (2017).

[54] A. Lewkowycz and O. Parrikar, J. High Energy Phys. 05 (2018) 147.

[55] A. C. Wall, Classical Quantum Gravity 31, 225007 (2014).

[56] A. May, J. High Energy Phys. 06 (2017) 118.

[57] P. Caputa, N. Kundu, M. Miyaji, T. Takayanagi, and K. Watanabe, J. High Energy Phys. 11 (2017) 097.

[58] J. Boruch, P. Caputa, and T. Takayanagi, Phys. Rev. D 103, 046017 (2021).

[59] J. Boruch, P. Caputa, D. Ge, and T. Takayanagi, J. High Energy Phys. 07 (2021) 016.

[60] P. Caputa and J. M. Magan, Phys. Rev. Lett. 122, 231302 (2019).

[61] H. A. Camargo, M. P. Heller, R. Jefferson, and J. Knaute, Phys. Rev. Lett. 123, 011601 (2019).

[62] H. Geng, Fortschr. Phys. 68, 2000036 (2020).

[63] A. R. Chandra, J. de Boer, M. Flory, M. P. Heller, S. Hörtner, and A. Rolph, J. High Energy Phys. 04 (2021) 207. 